\title{
Roberto Gordillo: planificador y líder, formador de profesionales y organizador gremial
}

\author{
Celso Martínez Musiño*
}

Artículo recibido:

5 de febrero de 2015

Artículo aceptado:

27 de septiembre de 2016

\section{Resumen}

La bibliotecología mexicana hace honor a sus precursores, es por ello que se presenta un análisis de la producción bibliográfica de Roberto Antonio Gordillo Gordillo. Aunque no es una biografía propiamente dicha, se hace un recuento general de la producción documental del notable profesional. El autor cuenta con 52 productos bibliográficos, de los cuales destacan ponencias, artículos y participaciones como directivo. Además, el profesor Gordillo fue formador de técnicos y profesionales, y fundador y afianzador de instituciones como la ahora Asociación Mexicana de Bibliotecarios A. C. y el Colegio Nacional de Bibliotecarios.

\footnotetext{
Dokumenta Consultoría e Integraciones, Mexico.

cmartinez@colmex.mx

INVESTIGACIÓN BIBLIOTECOLÓGICA, vol.32, núm.74, enero/marzo, 2018, México, ISSN: 2448-8321. pp. 123-143
} 
Se concluye que, con las aportaciones y trayectoria mencionadas, el profesor Gordillo es un baluarte en la bibliotecología nacional.

Palabras clave: Roberto Antonio Gordillo Gordillo; Bibliotecólogos mexicanos; Literatura bibliotecológica mexicana

Roberto Gordillo: planner, leader, teacher of professionals and union organizer

Celso Martínez-Musiño

\section{Abstract}

This analysis of the work of Professor Roberto Antonio Gordillo Gordillo is offered in the spirit of paying respect to the pioneers of Mexican Library Science. Not intended as a biography, this paper provides and overview of the writings and academic lectures of this important figure. Professor Gordillo was a notable teacher of professional library specialists and promoter of professional associations, including the Mexican Library Association and the National College of Librarians. In the history of Mexican library science, Professor Gordillo is a worthy representative and one of its keypillars.

Keywords: Roberto Gordillo Gordillo; Mexican librarians; Mexican librarian literature

\section{INTRODUCCIÓN}


aceptar el reto. Ante este hecho me planteé varias preguntas: ¿cómo puedo desarrollar un tema a partir de una persona que desconozco? ¿Cómo saber qué tan amplia es su trayectoria académica? ¿Qué actividades fundamentales realizó durante su vida? ¿Cómo mantuvo esas actividades, acaso fue por las relaciones académicas, políticas y por su habilidad para las relaciones públicas? ¿Cuál es la trascendencia de su obra, para qué, para quiénes? ¿Cómo responder estas preguntas? ¿A qué fuentes recurrir? ¿Cómo interpretar la información compilada? Se desarrolló así este estudio de tipo descriptivo, sin pretensiones de profundizar en la biografía del sujeto de estudio nos proponemos como objetivo general identificar y analizar la producción documental de Roberto Gordillo. El procedimiento de la investigación fue la búsqueda de información en catálogos de instituciones de educación superior como la ENBA, la UNAM y El Colegio de México, por mencionar algunos.

En el primer caso se localizaron 45 registros, en el segundo sólo se obtuvo un registro con 49 menciones. En el catálogo TESIUNAM se localizó un trabajo de Maestría en Bibliotecología y Estudios de la Información (Rojas Villarreal, 2009). Por su parte, en la base de datos CLASE no se obtuvieron resultados, lo mismo sucedió en Folletería mexicana. En El Colegio de México se localizaron tres registros: 1) Directorio de bibliotecas de la ciudad de México: una contribución a la VIII Feria Mexicana del Libro (Directory of Mexico City libraries: a contribution to the VIII Mexican Book Fair), compilado con Mary D. Parsons; 2) Una visión prospectiva de la profesión bibliotecaria en México (folleto), y 3) Una visión prospectiva de la profesión bibliotecaria en México (disco compacto). Para complementar las referencias y documentos se procedió a ejecutar búsquedas en Internet, de las cuales se desprenden 62 referencias en el buscador Google Scholar con la nomenclatura de búsqueda "Gordillo Gordillo" Roberto.

Es necesario aclarar que el documento "Puntos que deberán incluirse en la Ley General de Bibliotecas" no se incluye en este estudio porque, aunque se encontraba referenciado en la página del Foro Nacional de Armonización Bibliotecaria (FNALB, 2013), la liga no remite al documento respectivo. El artículo tampoco se encontró en las memorias de las XVIII Jornadas Mexicanas de Biblioteconomía. Después de localizar los datos bibliográficos se procedió a su análisis, a la redacción de los resultados y las conclusiones. El artículo se organiza de la manera siguiente: datos biográficos y época en la cual Roberto Gordillo desarrolló su obra, las principales aportaciones a la bibliotecología en México, y conclusiones. 


\section{Datos biográficos y ÉPOCA EN LA CUAL Roberto Gordillo DESARROLLÓ SU OBRA}

En distintas publicaciones se han vertido datos y testimonios de la biografía de Roberto Gordillo, por ejemplo, Estela Morales Campos le ha dedicado espacio en distintas publicaciones y eventos, como en la Memoria del IX Coloquio Internacional de Bibliotecarios (Morales Campos, 2004: 167-174) y en el libro Forjadores e impulsores de la bibliotecología latinoamericana (Morales Campos, 2006: 245-247). No obstante, dada la existencia de fuentes que abundan en la semblanza del personaje en cuestión, me detendré un tanto en la parte profesional, a partir de la década de 1940.

Roberto Gordillo nació el 12 de junio 1921 en Comitán, Chiapas, y no le fue ajeno el deseo de superación personal y profesional correspondiente con el proceso de reconstrucción social postrevolucionario, ingresando y preparándose en el naciente sistema educativo. Estudió en la Escuela Normal de Tuxtla Gutiérrez (1939-1942), en la Escuela Nacional de Bibliotecarios y Archivistas (1945-1947), y cursó la licenciatura en Educación (1947-1949) y la maestría en Biblioteconomía (1949-1950) en la Graduate School of Library Science en la University of Michigan (Morales Campos, 2006: 245), donde fue considerado como exalumno distinguido (Rojas Villarreal, 2009).

En relación a sus actividades laborales, en 1944 Roberto Gordillo trabajó en el Museo Regional de Antropología e Historia de Chiapas y en el Departamento de Bibliotecas del mismo estado. Entre sus actividades profesionales desempeñó el cargo de director de la Escuela Nacional de Biblioteconomía y Archivonomía, fue bibliotecario mayor del Instituto Tecnológico Autónomo de México, y en 1991 fue nombrado subdirector de Bibliotecas y Archivos de la Secretaría de Hacienda y Crédito Público. En 1993 presentó la iniciativa del Sistema Nacional de Bibliotecas Públicas. Falleció en 2009.

Me detendré un momento a describir algunos entornos socio-histórico-políticos, con el fin de situar de mejor manera la vida y aportaciones de nuestro autor. Durante sus estudios normalistas la Segunda Guerra Mundial estaba en pleno apogeo y México no fue ajeno a la conflagración. El país formó parte de la cadena de suministros de los Estados Unidos de América, además, trabajadores mexicanos fueron enviados a laborar en los campos de ese país. Por otra parte, en México se presentaba otro fenómeno, la inmigración del campo a las ciudades y la conformación de éstas como centros económicos y polos que marcaban la modernidad, en consecuencia, como un lugar que atraía a las poblaciones rurales y semirurales.

En su formación académica y desempeño profesional Roberto Gordillo vivió tiempos políticos en los cuales se comenzaba a formar un Estado nacional 
sin violencia armada de grandes proporciones. Políticamente, le tocaron periodos políticos representados por gobiernos de izquierda, centro, derecha, también llamados pendulares, representados por los presidentes Lázaro Cárdenas (1934-1940), Manuel Ávila Camacho (1940-1946), Miguel Alemán Valdés (1946-1952), Adolfo Ruiz Cortines (1952-1958), Adolfo López Mateos (19581964), Gustavo Díaz Ordaz (1964-1970), Luis Echeverría Álvarez (1970-1976), José López Portillo (1976-1982), Miguel de la Madrid (1982-1988), Carlos Salinas de Gortari (1988-1994), Ernesto Zedillo Ponce de León (1994-2000), Vicente Fox Quesada (2000-2006) y Felipe Calderón Hinojosa (2006-2012) (TPM, 2013).

Cada uno de los actores políticos tuvo una rúbrica distinta en la aplicación de políticas económicas, educativas y culturales. Por ejemplo, en el aspecto económico, desde mediados de la década de 1950 se incentivaba la industria nacional, mientras que a los productos extranjeros se les aplicaban impuestos altos, es decir, había medidas proteccionistas. Posteriormente hubo un proceso de apertura de los mercados internacionales, pasando de esta manera de un modelo de industrialización por sustitución de importaciones a una propuesta de transnacionalización del mercado interno. En cuando al aspecto educativo, se observó un proceso de formación docente y fortalecimiento de la educación pública; sin embargo, con la aparición de una pujante clase media también comenzó a consolidarse la educación privada. En relación al aspecto cultural y la necesidad de la creación de un Estado nacional que se apoyaba en un modelo educativo, se crearon instituciones como el Instituto Politécnico Nacional, El Colegio de México, la Facultad Latinoamericana de Ciencias Sociales (Flacso), la Universidad Autónoma Metropolitana y el Consejo Nacional para la Cultura y las Artes, por mencionar algunas.

La conjunción de factores sociales, económicos y culturales dio paso a la necesidad de crear políticas que atendieran individuos y colectivos demandantes de servicios culturales en general y particulares, como los que ofrecen las bibliotecas. Bien vale la pena detenerse para saber cómo han ido desarrollándose esas políticas y cómo se han reflejado en relación a la cantidad de bibliotecas establecidas, así como las colecciones y sus servicios. A mediados de la década de 1960 había 837 bibliotecas cuyo total de volúmenes era de 6112910 (no se menciona la cantidad de usuarios) para atender a una población total en México de 44145000 habitantes (Gordillo Gordillo, 1971a: tabla anexa).

Posteriormente, en un estudio estadístico crítico de Martínez Musiño, Cruz Linares y Martínez Guerrero (2013: 44) con datos del Instituto Nacional de Estadística y Geografía, encontramos que en 1990 había ya una infraestructura bibliotecaria consolidada por 6964 bibliotecas con un acervo 
de 24.9 millones de volúmenes y 89.5 millones de servicios [sic]. A partir de 2008 se reducen significativamente las consultas promedio, casi en 40 millones. El año con mayor cantidad de consultas fue 2003 con 134.1 millones, mientras que en 2010 las consultas promedio se redujeron a menos de la mitad, 64.3 millones. A partir de entonces, se puede observar que el acrecentamiento gradual de bibliotecas y acervos no tiene una relación directa con las consultas promedio, sino que se da una relación inversa porque estas últimas han disminuido.

Al conjuntar algunos aspectos mínimos de la época en la que Gordillo Gordillo vivió y desarrolló su obra podemos apreciar una gran cantidad de cambios y factores económicos, históricos, educativos, culturales y políticos. La descripción de estos aspectos deja mucho para poder profundizar en futuras ocasiones.

\section{Principales aportaciones de Roberto Gordillo a la bibliotecología en México}

Al observar el contexto histórico en el cual Roberto Gordillo se desarrolló académica y profesionalmente, los problemas que trató de resolver son los aspectos de profesionalización del personal educativo (docencia) (Gordillo Gordillo, 1960b; 1985e; 1979), la consolidación de instituciones de profesionales, profesional (directivo) y el establecimiento y consolidación de organismos gremiales. Para abundar en el contexto histórico de la bibliotecología en México se recomienda la tesis Testimonios de la bibliotecología mexicana: educación 1915-1945 (Morales Campos, 1987), donde se exponen los antecedentes, los planes de estudio y la influencia europea y norteamericana, la conformación de la Escuela Nacional de Bibliotecarios y Archivistas, y la situación de las bibliotecas y los bibliotecarios. El estudio se desarrolló con base en investigación documental y la participación de informantes.

\section{Roberto Gordillo como formador}

Con formación normalista y especializaciones en el extranjero en educación y en biblioteconomía, Roberto Gordillo dedicó mucho de su tiempo a la docencia y a la creación de espacios para la consolidación de nuevas organizaciones educativas. Por ejemplo: 
En dos periodos (de 1954 a 1956 y de 1969 a 1982) ejerció la docencia en la Escuela Nacional de Biblioteconomía y Archivonomía (ENBA) de la Secretaría de Educación Pública (en el primero, aún llevaba el nombre de Escuela Nacional de Bibliotecarios y Archivistas). Ha participado como profesor en un sinnúmero de cursos, talleres y seminarios nacionales, en temas como: Selección, Legislación, Metodología de la investigación, Administración de bibliotecas universitarias, etcétera. Igualmente, ha sido director de una gran cantidad de tesis de nivel licenciatura. (Morales Campos, 2006: 245)

Además de la docencia, también fue un impulsor para el establecimiento de nuevas instituciones educativas. En Chiapas, por ejemplo, durante las XXII Jornadas Mexicanas de Biblioteconomía

se le rindió homenaje al Maestro Roberto Gordillo, originario de Comitán Chiapas. En dicho acto se le reconoció por sus 47 años de labor sobresaliente, de compromiso indeclinable hacia el servicio bibliotecario mexicano. En su discurso el profesor Gordillo propuso a las autoridades de la Universidad Autónoma y al Gobierno del Estado de Chiapas, la posibilidad de presentar un proyecto para establecer la carrera de la Licenciatura en Biblioteconomía en la Universidad. La propuesta fue aprobada [...] (Chacón Escobar, 2009: 244)

El profesor Gordillo se dedicó, pues, a preparar alumnos, impulsar instituciones formadoras de bibliotecarios y ocupó puestos directivos; me permito hacer algunas preguntas para futuras investigaciones: ¿quiénes lo conocieron como docente? ¿Es posible aún entrevistarlos? ¿Imponía alguna teoría o escuela administrativa? ¿Cómo era como jefe? ¿Qué tipo de liderazgo ejercía? ¿Es posible contactar a sus compañeros de trabajo? ¿Qué herramientas de levantamiento de datos e información serían las adecuadas? ¿Es posible obtener más información al respecto en otros documentos escritos, o mediante el análisis de grabaciones de entrevistas que le hayan hecho?

\section{Roberto Gordillo como autor}

El profesor Gordillo es autor de 52 documentos, entre artículos, ensayos, informes, textos de homenajes y respuestas a homenajes, por mencionar algunos, en los cuales firma en su gran mayoría de manera individual. En dos ocasiones se identificaron textos con rúbrica colectiva (Parsons y Gordillo Gordillo, 1958; Feria Basurto, Gordillo Gordillo y Palacios Beltrán, 1984-1985). Los primeros y el último texto identificados en catálogos, bases de datos e Internet datan de 1957 (Gordillo Gordillo, 1957a; 1957b; 1957c y 1957d) y 2006 (Gordillo Gordillo, 2006), respectivamente. En cuanto al 
tipo de documentos en los que publicó destacan los impresos Boletín de la Escuela Nacional de Bibliotecarios y Archivistas, y el Boletín de la Asociación Mexicana de Bibliotecarios, las memorias de las Jornadas Mexicanas de Biblioteconomia, Bibliografía y Canje, las Jornadas Mexicanas de Biblioteconomía y Semana de bibliotecología. Hasta la fecha en la que se realizó la búsqueda de la producción bibliográfica impresa se localizaron 52 documentos; según su distribución por décadas, se puede decir que las más prolíficas fueron 1980 y 1960. Hay que mencionar también la publicación de tres libros: Notas sobre administración y organización de bibliotecas para instituciones de enseñanza superior, Una visión prospectiva de la profesión bibliotecaria en México y el Directorio de bibliotecas de la Ciudad de México = Directory of Mexico City Libraries (Figura 1).

\section{Boletines}

Bibliotecas y Archivos (9 documentos)

Boletín de la Asociación Mexicana de Bibliotecarios (1 documento)

Boletín de la Escuela Nacional de Bibliotecarios y Archivistas (8 documentos)

Memorias de congresos

Organizadas por la AMBAC (26 documentos)

Semana de bibliotecología (2 documentos)

IX Coloquio Internacional de Bibliotecarios. Guadalajara (1 documento)

\section{Revistas académicas}

Ciencia bibliotecaria (1 documento)

Libros (3 documentos)

CD/ROM (1 documento)

Figura 1. Publicaciones de Roberto Gordillo (localizadas y referenciadas en esta investigación) Fuente: elaboración propia

Nota: para este estudio no se consideraron las participaciones de Roberto Gordillo en el Noticiero de la AMBAC 
Ahora bien, ¿cuáles son los temas abordados por el autor? Con fines de organización se describirán de acuerdo a sus actividades principales: funciones y servicios de la biblioteca (Gordillo Gordillo, 1983a), como director de instituciones, intereses de participación gremial, los distintos tipos de bibliotecas, la legislación bibliotecaria, y tópicos diversos.

a) De acuerdo a las funciones y servicios de la biblioteca destacan los temas de planeamiento, la selección de libros, los servicios bibliotecarios, información y consulta. Como profesional que laboró en las bibliotecas no se enfocó en los procesos técnicos; sin embargo, sí se interesó en el tema de la bibliografía, sobre todo la nacional (Gordillo Gordillo, 1958). Por otra parte, los usuarios fueron temas de interés (Gordillo Gordillo, 1989a), en especial los profesores y los marginales (Gordillo Gordillo, 1996). Si consideramos breve y gráficamente las funciones de las bibliotecas, resulta necesaria una Dirección que coordine las actividades para la Entrada de las colecciones y su posterior Organización, y finalmente la Salida de las mismas mediante la oferta de servicios. Roberto Godillo participó en casi todos los tópicos, con excepción de la organización de las colecciones (Figura 2).

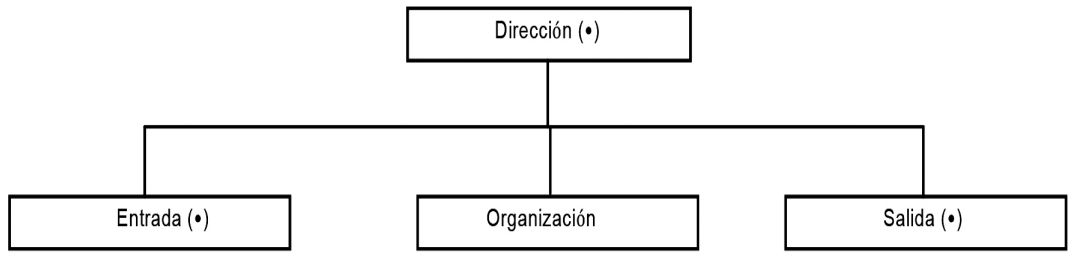

Figura 2. Cobertura temática del profesor Gordillo en la descripción de las funciones de las bibliotecas

Fuente: elaboración propia

Nota. El símbolo $(\bullet)$ representa las áreas temáticas tratadas por Roberto Gordillo

Para finalizar este apartado, me referiré a las palabras de Adolfo Rodríguez Gallardo con motivo del 25 aniversario del Colegio Nacional de Bibliotecarios:

Roberto Gordillo cuenta con una amplia bibliografía sobre distintos asuntos relacionados con la bibliotecología mexicana, ha abordado una vasta temática tanto sobre aspectos de servicio, como de políticas gubernamentales sobre información, o la educación en nuestra disciplina, el papel de los bibliotecarios ante los servicios y el rol que estos desempeñan en la vida cultural y educativa de la nación. (Rodríguez Gallardo, 2006: 3) 
b) El país requería de personas preparadas, no sólo profesionales sino especialistas en temas específicos como la biblioteconomía. El profesor Gordillo fue testigo del desarrollo de distintas escuelas y teorías administrativas y el desarrollo de la ciencia de la información. Desde el enfoque administrativo se desarrollaron las teorías de la administración científica, la administración general, la teoría administrativa del comportamiento, la teoría de la ciencia administrativa hasta la teoría del desarrollo organizacional. Por otro lado, desde la ciencia de la información vivió el periodo del nacimiento de la documentación, la información científica, la recuperación de la información, el nacimiento de la ciencia de la información, la informática y la bibliometría (Figura 3).

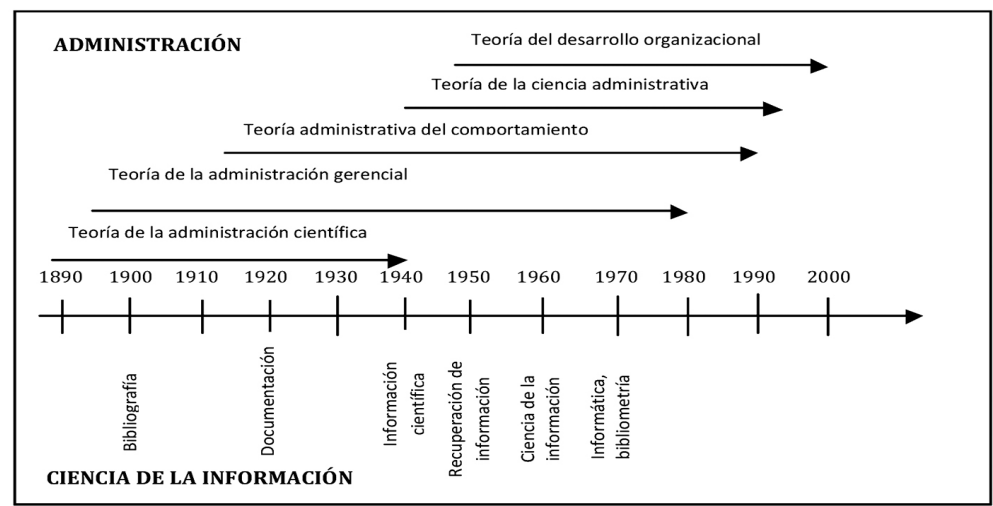

Figura 3. Línea del tiempo de la evolución de la teoría administrativa y la ciencia de la información

Fuente: Martínez Musiño (2013) con los siguientes datos: para la teoría administrativa, Jones y George (2008) y para la ciencia de la información, Schrader (1984)

Ante los cambios vistos por Roberto Gordillo, vale la pena preguntarnos para futuras investigaciones: ¿cómo concibió los cambios de los enfoques administrativos y de qué manera los llevó al salón de clases? ¿Cómo enlazó las teorías administrativas en su quehacer profesional? ¿De qué manera se influenciaron los cambios de la ciencia de la información en la biblioteconomía? ¿Es posible identificar esos cambios en sus escritos? A grandes rasgos podemos adelantar que sí, Gordillo abordó los tópicos del planeamiento (Gordillo Gordillo, 1981), la administración (Gordillo Gordillo, 1980b), y la evaluación de los servicios bibliotecarios (Gordillo Gordillo, 1980a), o el análisis de colegas cuyo trabajo se refería a la filosofía de los servicios de información (Gordillo Gordillo, 1975a).

c) En cuanto a su participación gremial destacan las publicaciones relacionadas con los informes del Colegio Nacional de Bibliotecarios (Gordillo 
Gordillo, 1982) y la necesidad de actualizaciones al reglamento de la Asociación Mexicana de Bibliotecarios (Gordillo Gordillo, 1975c). Desde las Primeras Jornadas Mexicanas de Biblioteconomía, Bibliografía y Canje, Roberto Gordillo, como líder de organizaciones gremiales, era capaz de convocar personalidades y autoridades de primer nivel. Por ejemplo, cuando era secretario de la Asociación Mexicana de Bibliotecarios participó en la organización de las Primeras Jornadas Mexicanas de Biblioteconomía, Bibliografía y Canje, del 2 al 7 de diciembre de 1957. A las 20:00 del domingo 2 se inauguró el evento en la Sala Manuel M. Ponce del Palacio de Bellas Artes, acudieron José Ángel Ceniceros, secretario de Educación Pública, y Nabor Carrillo Flores, rector de la UNAM. En estas jornadas participaron María Luisa Ocampo (jefa del Departamento de Bibliotecas de la SEP), Tobías Chávez (profesor de Biblioteconomía en la UNAM), Ignacio Medina (Departamento de Distrito Federal), Manuel Alcalá Anaya (director de la Biblioteca Nacional), Armando M. Sandoval, Rafael Carrasco Puente (director de la Hemeroteca Nacional), Carlos A. Madrazo (ENBA), Fidencio Salazar Reyes, Roberto A. Gordillo (AMBAC, subdirector y jefe del Departamento de consulta de la Biblioteca del Mexico City College), Alicia Perales, Jovita T. de Zubaran, María Teresa Chávez, José Ignacio Mantecón, Juan B. Iguíniz y Pedro Zamora, por mencionar algunas autoridades y personalidades del ámbito bibliotecario.

En este evento Roberto Gordillo participó con las ponencias "Análisis y comentarios basados en los cuestionarios para el directorio de bibliotecas de la República Mexicana" (Gordillo Gordillo, 1957a), "Libre acceso del lector a los estantes" (Gordillo Gordillo, 1957b), "Selección de libros" (Gordillo Gordillo, 1957c) y "Servicio de información y de consulta" (Gordillo Gordi1lo, 1957d). Su labor en eventos académicos es y ha sido reconocida aún después de su deceso, tanto por la institución donde impartió clases como por aquellos gremios que erigió y que posteriormente se consolidaron.

d) En relación al interés de Roberto Gordillo por los temas relacionados por distintos tipos de bibliotecas, se pueden mencionar los siguientes documentos: "Las bibliotecas públicas de la República Mexicana: análisis de su situación actual" (Gordillo Gordillo, 1971b), "Normas para el establecimiento y sostenimiento de bibliotecas públicas en la República Mexicana" (Gordillo Gordillo, 1977), "Reflexiones sobre PRODENASBI y revisión de la propuesta de normas para el establecimiento y sostenimiento de bibliotecas públicas en la República Mexicana” (Gordillo Gordillo, 1983b), "Mesa redonda sobre la Biblioteca Pública que se necesita en México” (Feria Basurto, Gordillo Gordillo y Palacios Beltrán, 1984-1985), “El usuario marginado: el caso de las escuelas y facultades de psicología de la República Mexicana en relaciones con sus recursos de información” (Gordillo Gordillo, 1985c), 
"El profesor universitario como usuario y promotor del servicio bibliotecario: análisis de dos casos" (Gordillo Gordillo, 1985b), "La bibliografía nacional” (Gordillo Gordillo, 1959a), "El aspecto técnico: la bibliografía nacional” (Gordillo Gordillo, 1967a) y, finalmente, el artículo "Normas mínimas para el servicio bibliotecario en universidades latinoamericanas" (Gordillo Gordillo, 1975b). En conjunto, este grupo de escritos representa un conocimiento amplio de los distintos tipos de bibliotecas (públicas, universitarias, nacionales y especializadas) desde su normatividad, sus usuarios y su funcionamiento.

e) Roberto Gordillo participó activamente para la propuesta de la normatividad bibliotecaria y de su legislación (Gordillo Gordillo, 1974; 1975b; 1977; 1989b y 1994). Respecto a este tema, si la construcción de la legislación requiere de una etapa prelegislativa en la cual es necesario un diagnóstico en el que se responden preguntas como ¿para qué se debe aplicar una ley?, ¿cuáles son los escenarios posibles de trabajo?, ¿qué actores deben involucrarse en el diseño de la propuesta?, y la consecuente presentación de alternativas a las autoridades correspondientes (Mora Donato, 2013), el maestro Gordillo observó bastante bien la dinámica política en la cual había que adoptar un lenguaje propio de los bibliotecarios y la descripción y adopción de normas bibliotecarias para obtener un posicionamiento gremial.

f) En los temas diversos encontramos la prospección de las instituciones formadoras de profesionales (Gordillo Gordillo, 1985d), de la profesión bibliotecaria (Gordillo Gordillo, 2006), del libro y su cuidado (Gordillo Gordillo, 1993), la ética profesional (Gordillo Gordillo, 1986), la misión de las bibliotecas en comunidades específicas (Gordillo Gordillo, 1988), la necesidad de adherirse a la Asociación (Gordillo Gordillo, 1998), la necesidad de atender los servicios bibliotecarios en instituciones de educación básica, y la vinculación de los servicios con los materiales de lectura para los recién alfabetizados (Gordillo Gordillo, 1967b). Desde su participación en las Terceras Jornadas de Biblioteconomía inauguradas por Jaime Torres Bodet y en las cuales participaron bibliotecarios del continente americano, el maestro Gordillo presentó como propósitos "[...] analizar el servicio bibliotecario en las escuelas primarias y secundarias y a sugerir, [... la forma más adecuada para lograr el establecimiento [de bibliotecas] donde no existan [...]" (Gordillo Gordi1lo, 1960c: ix).

Por otra parte, cabe destacar aquellos documentos relacionados con el quehacer docente (Gordillo Gordillo, 1984), informes institucionales (Gordillo Gordillo, 1959c; 1960a; 1960-1961; 1961-1962a; 1961-1962b; 1961-1962c; 1962-1963a), e incluso aspectos extrainstitucionales tales como la dedicación de algunas palabras en relación al deceso de Agustín Hernández Piuki el 12 de octubre de 1962 (Gordillo Gordillo, 1962-1963c). 


\section{Roberto Gordillo como organizador gremial}

La suma de voluntades fue otro de los elementos que había que atender. El resultado de este interés fue la erección de asociaciones no profesionales y profesionales de agremiados bibliotecarios. La profesionalización y la adopción de normas eran una condición para el mejoramiento de las relaciones y la consolidación de las instituciones formadoras; sin embargo, para tener éxito en las gestiones hay que hacer política y uno de esos espacios lo ganan los gremios organizados.

El establecimiento de una asociación puede resumirse de la siguiente manera: a) identificar la necesidad de asociarse; b) identificar y seleccionar qué tipo de representación legal se requiere (S. C., A. C., etc.); c) invitar y convencer a colegas; d) describir y definir el uso social de la asociación; e) determinar quién paga y quién o quiénes realizarán el trámite de registro; f) ya registrada la asociación, cómo invitar y convencer a los bibliotecarios a asociarse. A Roberto Gordillo le correspondió participar en estos procesos en dos ocasiones. La primera, para la creación de la Asociación Mexicana de Bibliotecarios, cuya rúbrica se encuentra en el acta constitutiva publicada en Internet (AMBAC, 2013); la segunda, en la conformación del Colegio Nacional de Bibliotecarios, cuyo reconocimiento se encuentra disponible en la página de Internet (CNB, 2013). Roberto Gordillo presidió la AMBAC los periodos 1960-1961, 1964-1965 y 1987-1989, fue declarado socio honorario de la misma en 1982 con base en sus siete años de servicio (Rojas Villarreal, 2009). Además de dirigir esta asociación, en periodos que no tenía estricta adhesión como directivo, Gordillo Gordillo opinaba y reflexionaba respecto al quehacer de los expresidentes de la AMBAC (Gordillo Gordillo, 1985a), o bien del desarrollo de las bibliotecas e instituciones formadoras de profesionales bibliotecarios (Gordillo Gordillo, 2004).

Al maestro Gordillo "[...] le ha gustado ser el primero en muchos aspectos y sus colegas lo han aceptado así: primer presidente de la Asociación Mexicana de Bibliotecarios, A.C., primer presidente del Colegio Nacional de Bibliotecarios, A.C....", como lo menciona Sergio López Ruelas en la Memoria del IX Coloquio Internacional de Bibliotecarios (López Ruelas, 2004: 165). Además de la AMBAC y el CNB "promovió la creación de la hoy desaparecida ABIESI” (Rodríguez Gallardo, 2006: 2). Para profundizar en la historia de la AMBAC y la dinámica de sus relaciones gremiales se recomienda la lectura de la monografía La Asociación Mexicana de Bibliotecarios, A.C.: notas para su bistoria (Fernández de Zamora, 1995), la tesis La bibliotecología y el asocianismo en México: la Asociación Mexicana de Bibliotecarios A. C. (Rojas Villarreal, 2009), cuyo autor indaga los antecedentes gremiales desde 1924, 
la estructura, finanzas y la forma de gobierno de las asociaciones, así como la descripción de sus actividades, publicaciones y la relación con otro tipo de instituciones, y el artículo "La AMBAC en la historia, una visión gráfica: las Jornadas Mexicanas de Biblioteconomía" (España González, 2015). Esta autora hace mención al nombramiento de Gordillo Gordillo como socio honorario "por su destacada trayectoria y por la imborrable huella que dejó para la profesión bibliotecaria” (España González, 2015: 52) en las $13^{a}$ Jornadas Mexicanas de Biblioteconomía.

Roberto Gordillo no sólo fue organizador gremial, sino que también contribuyó en la conciencia misma de su desempeño; así lo expresa en su ensayo "La afiliación a las asociaciones de bibliotecarios: el caso de la pertinencia y de la pertenencia": "[la] satisfacción del deseo de pertenecer a un grupo social" (Gordillo Gordillo, 1998: 15). Por otro lado, con motivo del 25 aniversario del Colegio Nacional de Bibliotecarios, expresaba la necesidad de incrementar la membresía, argumentando que de 1987 a 2003 sólo se había alcanzado la cantidad de 107 socios, lo cual representaba en ese entonces el $5 \%$ de titulados, aproximadamente (Gordillo Gordillo, 2006: 6).

Ante la preocupación del propio Gordillo Gordillo por la representatividad gremial de las asociaciones, vale la pena preguntarnos si éstas han tenido un impacto -y en qué medida- como interlocutoras con actores políticos y sociales. Acaso es posible medir esa intermediación con gremios de abogados, contadores, ingenieros y economistas, por mencionar algunos. Tal vez estas preguntas no tengan respuesta en estos momentos, pero pueden considerarse para futuros estudios.

Para cerrar este apartado, en una entrevista previa al seminario Fuentes Históricas de la Bibliotecología en México, en septiembre de 2013, Julio Zetter Leal, presidente de la Academia Mexicana de Bibliografía, habló acerca de las actividades directivas gremiales y de Roberto Gordillo. Al preguntarle Celso Martínez Musiño acerca de qué es una asociación, respondió que "es una organización con una estructura que conjunta a personas que comulgan con una meta común [...] organización profesional de académicos para su fortalecimiento [...], cuyo objetivo es desarrollar desde una perspectiva académica el aspecto relacionado con los libros, las bibliotecas, los documentos y la actividad bibliotecaria [...] e ir más allá [...]”.

Respecto a su percepción de Roberto Gordillo, se expresó de él como "un líder de opinión, como un líder profesional de las bibliotecas [...] como una persona apasionada, con convicciones [...] formó muchos cuadros como docente, marcó un camino y dejó huella [...] construyó y cimentó un camino [...] para que las generaciones continúen". 
Dada su trayectoria, el profesor Gordillo ha sido objeto de numerosos reconocimientos y homenajes. Entre ellos destacan el video preparado por el Instituto Mexicano de la Administración del Conocimiento (IMAC, 2013), el homenaje organizado por el Colegio Nacional de Bibliotecarios, el homenaje en el IX Coloquio Internacional de Bibliotecarios, Guadalajara, en el cual participaron Sergio López Ruelas (López Ruelas, 2004), María Luisa Armendáriz (Armendáriz, 2004) y Estela Morales Campos (Morales Campos, 2004). Morales Campos también le dedicó un apartado en su libro Forjadores e impulsores de la bibliotecología latinoamericana (Morales Campos, 2006) y Adolfo Rodríguez Gallardo (Rodríguez Gallardo, 2006) incluyó una reseña sobre su vida y obra en el disco compacto Una visión prospectiva de la profesión bibliotecaria en México, editado por el Colegio Nacional de Bibliotecarios, que mantiene a su vez a Roberto Gordillo en un lugar privilegiado en su página de Internet como su fundador (CNB, 2013). Además, en el portal de Internet de la Asociación Mexicana de Bibliotecarios se puede consultar el acta constitutiva y la rúbrica del maestro Gordillo.

\section{Conclusiones}

Al investigar la producción documental de Roberto Gordillo se identificaron más de 50 escritos publicados básicamente en memorias de congresos y boletines de una institución formadora de bibliotecarios. En menor medida se localizaron participaciones en libros y revistas académicas. Por otro lado, en relación a los contenidos, sin llegar a un nivel de profundidad, es posible dilucidar que Gordillo Gordillo invirtió tiempo y dedicación a múltiples contenidos, entre ellos la normatividad de las bibliotecas y las funciones y servicios de las mismas. Identificó, además, la importancia del usuario de las bibliotecas y la orientación de las metas y objetivos para su atención y satisfacción. Por otro lado, es posible encontrar en algunos documentos la aplicación de las etapas administrativas de planeación, organización, dirección y control.

Roberto Antonio Gordillo Gordillo sobresale como docente, directivo de unidades de información, organizador gremial y generador de conocimientos. Como profesor, es recordado y estimado por muchas generaciones de profesionales de la información mientras que, como profesional, destacó en diversas instituciones públicas y privadas. Se le reconoce como convocante de colegas y por conformar instituciones gremiales que permanecen hasta nuestros días. Se distingue además por dejar un legado escrito en el cual abarcó diversidad de temas, tanto de las bibliotecas como de las colecciones y los servicios bibliotecarios, y de la información. Los bibliotecarios 
y sus asociaciones también fueron de interés. La normatividad de las bibliotecas y la legislación fueron temas logrados, tanto en sus escritos como en consecuencias tangibles, por ejemplo, incidir en aquellos que tomaban las decisiones. La información y los archivos también se distinguen en su legado documental.

Si se pudiera sintetizar la aportación de esta investigación -aún no terminada- es la compilación de los documentos producidos por Roberto Gordi1lo. Sin embargo, para futuras investigaciones, es posible profundizar en los variados temas que le interesaron. Por otra parte, es sugerente indagar con aquellas personas que lo conocieron en alguna de las distintas facetas (profesor, profesional, directivo), o en su defecto investigar en archivos de las instituciones en las que laboró o en las instituciones que el formó y consolidó.

\section{REFERENCIAS}

Armendáriz, M. L. 2004. "Homenaje a Roberto Antonio Gordillo Gordillo", en Vinculación de las bibliotecas y la academia: un esfuerzo compartido: Memoria del IX Coloquio Internacional de Bibliotecarios. Guadalajara, 175-176. México: Universidad de Guadalajara. Fecha de consulta: 18 de agosto de 2016. http://rebiudg.udg.mx/coloquio/docs/memorias/IX_CIB_2002.pdf

AMBAC (Asociación Mexicana de Bibliotecarios A. C.). 2013. "Acta constitutiva AMBAC”. Portal AMBAC. México: AMBAC. Fecha de consulta: 18 de agosto de 2016. http://www.ambac.org.mx/normatividad/acta_constitutiva_AMBAC.pdf

Chacón Escobar, R. E. 2009. "La formación de recursos humanos en Bibliotecología en la Universidad Autónoma de Chiapas: 1992-2008”, en Memoria del Octavo Congreso Nacional de Bibliotecas Públicas: a 25 años de la Red Nacional de Bibliotecas Públicas y a 20 años de la promulgación de la Ley General de Bibliotecas: Tuxtla Gutiérrez, Chiapas, del 9 al 11 de octubre de 2008, 241-249. México, Gobierno Federal: Conaculta. Fecha de consulta: 18 de agosto de 2016. http://dgb.conaculta.gob.mx/Documentos/PublicacionesDGB/ColeccionMemorias/Memoria8Congreso.pdf

CNB (Colegio Nacional de Bibliotecarios). 2013. "Presidente y expresidentes", en Portal CNB. México: CNB. Fecha de consulta: 19 de agosto de 2016. http://www. cnb.org.mx/index.php/acerca-cnb/presidente-y-expresidentes

España González, M. E. 2015. "La AMBAC en la historia, una visión gráfica: las Jornadas Mexicanas de Biblioteconomía”, en Memoria: tecnología de la información; tendencias e impacto en las bibliotecas, Saúl Armendáriz Sánchez, comp. 50-59. México: AMBAC. Fecha de consulta: 19 de agosto de 2016. http://www.ambac. org.mx/pub/jmb/pdf/45jmb.pdf

Fernández de Zamora, R. M. 1995. La Asociación Mexicana de Bibliotecarios, A.C.: notas para su bistoria. México: AMBAC.

FNALB (Foro Nacional de Armonización Bibliotecaria). 2013. Doctrina. México. Fecha de consulta: 13 de mayo de 2015. http://www.fnalb.org.mx/styled-5/ styled-11/index.html 
IMAC (Instituto Mexicano de la Administración del Conocimiento). 2013. Homenaje al Mtro. Roberto Antonio Gordillo Gordillo, video. México: IMAC. Fecha de consulta: 19 de agosto de2016. https://www.youtube.com/watch?v=NZsv-Skblbg

Jones, G. R. y J. M. George. 2008. Contemporary management, 5a ed. Boston: McGraw-Hill/ Irwin.

López Ruelas, S. 2004. "Homenaje al bibliotecario: Roberto Antonio Gordillo Gordillo", en Vinculación de las bibliotecas y la academia: un esfuerzo compartido: memoria del IX Coloquio Internacional de Bibliotecarios, 163-166. Guadalajara, México: Universidad de Guadalajara. Fecha de consulta: 19 de agosto de 2016. http://rebiudg.udg.mx/coloquio/docs/memorias/IX_CIB_2002.pdf

Martínez Musiño, C. 2013. "La información en la disciplina administrativa". E-ciencias de la información 3 (2): 1-15. Fecha de consulta: 19 de agosto de 2016. http:// revistas.ucr.ac.cr/index.php/eciencias/article/download/10655/10051

Martínez Musiño, C., G. Cruz Linares y R. M. Martínez Guerrero. 2013. "Un estudio estadístico crítico de los usuarios de las bibliotecas en México: 1990-2010”. Crítica Bibliotecológica 6 (2): 41-49. Fecha de consulta: 19 de agosto de 2016. http:// eprints.rclis.org/23556/1/c.b.2013.vol.6.no.2_martinez-musi\%C3\%B1o.pdf

Mora Donato, C. 2013. "Modelo para la construcción de normas: escenario posible de trabajo", conferencia presentada en Reformas al marco jurídico de las bibliotecas en México. Legislación bibliotecaria en México. Foro Nacional de Armonización Legislativa Bibliotecaria, 19 de junio de 2013. México.

Morales Campos, E. 1987. Testimonio de la Bibliotecología mexicana: educación: 19151954. México. Fecha de consulta: 19 de agosto de 2016. http://132.248.9.195/ ptb2010/anteriores/0056501/Index.html

Morales Campos, E. 2004. "Don Roberto Gordillo, el bibliotecario, el formador de jóvenes", en Vinculación de las bibliotecas y la academia: un esfuerzo compartido: memoria del IX Coloquio Internacional de Bibliotecarios, María de los Ángeles River, comp., 167-174. Guadalajara, México: Universidad de Guadalajara. Fecha de consulta: 19 de agosto de 2016. http://rebiudg.udg.mx/coloquio/docs/memorias/IX_CIB_2002.pdf

Morales Campos, E. 2006. "Gordillo Gordillo, Roberto Antonio", en Forjadores e impulsores de la bibliotecología latinoamericana, Estela Morales Campos, 245-247. México: UNAM, Centro Universitario de Investigaciones Bibliotecológicas, Dirección General de Bibliotecas. Fecha de consulta: 19 de agosto de 2016. http://132.248.242.6/ publica/conmutarl.php? arch=1\&idx=177»idx=177

Rodríguez Gallardo, A. 2006. "Mtro. Roberto Gordillo Gordillo: reseña curricular”, en Una visión prospectiva de la profesión bibliotecaria en México, disco compacto. México: CNB.

Rojas Villarreal, M. J. 2009. "La bibliotecología y el asocianismo en México: la Asociación Mexicana de Bibliotecarios A.C.”. Tesis de Maestría en Bibliotecología y Estudios de la Información, UNAM, FFyL-CUIB. Fecha de consulta: 13 de mayo de 2015. http://132.248.9.195/ptd2009/febrero/0639939/0639939_A1.pdf\#search=gordillo gordillo roberto

Schrader, A. M. 1984. "In search of a name: information science and its conceptual antecedents". Library and Information Science Research, an International Journal 6 (3): $227-271$. 
TPM (Todos los presidentes de México). 2013. Presidentes de México. México. Fecha de consulta: 13 de mayo de 2015. http://presidentes.mx/

\section{Referencias de Roberto Antonio Gordillo Gordillo}

Feria Basurto, L., R. A. Gordillo Gordillo y M. Palacios Beltrán. 1984-1985. “Mesa redonda sobre la Biblioteca Pública que se necesita en México”, en XV Jornadas Mexicanas de Biblioteconomía, del 30 de abril al 4 de Mayo de 1984, Tlaxcala, Tlaxcala: memorias, 375-386. México: AMBAC.

Gordillo Gordillo, R. A. 1957a. "Análisis y comentarios basados en los cuestionarios para el directorio de bibliotecas de la República Mexicana”, en Jornadas Mexicanas de Biblioteconomía, Bibliografía y Canje, 51-83. Memorias. México: AMBAC. (1a. ed. 1956, Cd. México)

Gordillo Gordillo, R. A. 1957b. "Libre acceso del lector a los estantes”, en Jornadas Mexicanas de Biblioteconomía, Bibliografía y Canje, 102-106. Memorias. México: AMBAC. (1a. ed. 1956, Cd. México)

Gordillo Gordillo, R. A. 1957c. "Selección de libros", en Jornadas Mexicanas de Biblioteconomia, Bibliografía y Canje, 249-253. Memorias. México: AMBAC. (1a. ed. 1956, Cd. México)

Gordillo Gordillo, R. A. 1957d. "Servicio de información y de consulta”, en Jornadas Mexicanas de Biblioteconomía, Bibliografía y Canje, 84-89. Memorias. México: AMBAC. (1a. ed. 1956, Cd. México)

Gordillo Gordillo, R. A. 1958. "La bibliografía mexicana del siglo XX: posibilidades de mejorar su compilación”. Boletín de la Asociación Mexicana de Bibliotecarios 1 (2-3): 61-66.

Gordillo Gordillo, R. A. 1959a. "La bibliografía nacional”, en II Jornadas Mexicanas de Biblioteconomía, Bibliografía y Canje, 12 al 15 de abril de 1959, San Luis Potosí, S.L.P.: memorias, 68-78. México: Centro de Documentación Científica y Técnica de México.

Gordillo Gordillo, R. A. 1959b. Notas sobre administración y organización de bibliotecas para instituciones de enseñanza superior. México: SEP, Departamento de Bibliotecas.

Gordillo Gordillo, R. A. 1959c. "Palabras de introducción a la ceremonia". Boletín de la Escuela Nacional de Bibliotecarios y Archivistas 4 (11-12) (septiembre-octubre): 11-12.

Gordillo Gordillo, R. A. 1960a. "Balance de XV años: palabras por el profesor Roberto A. Gordillo, Director de la Escuela”. Boletín de la Escuela Nacional de Bibliotecarios y Archivistas 4 (16-21) (abril-septiembre): 19-25.

Gordillo Gordillo, R. A. 1960b. "Preparación profesional del profesor bibliotecario", en Jornadas Mexicanas de Biblioteconomía, 67-72. Memorias. México: AMBAC. (3a.ed. 1960, Cd. México)

Gordillo Gordillo, R. A. 1960c. "Propósitos de las Terceras Jornadas", en Terceras Jornadas de Biblioteconomía del 5 al 8 de Diciembre de 1960, VII-XI. Memorias. México: AMBAC.

Gordillo Gordillo, R. A. 1960-1961. "Resumen del ciclo de conferencias". Boletín de la Escuela Nacional de Bibliotecarios y Archivistas 4 (22-27) (septiembre-marzo): 29-36. 
Gordillo Gordillo, R. A. 1961-1962a. “[Décimo séptimo] XVII aniversario”. Boletín de la Escuela Nacional de Bibliotecarios y Archivistas 5 (28) (abril-diciembre, enero-agosto): 73-75.

Gordillo Gordillo, R. A. 1961-1962b. "Informe del profesor Roberto A. Gordillo, director de la escuela". Boletín de la Escuela Nacional de Bibliotecarios y Archivistas 5 (28) (abril-diciembre, enero-agosto): 85-95.

Gordillo Gordillo, R. A. 1961-1962c. "Palabras del profesor Roberto A. Gordillo, Director de la Escuela”. Boletín de la Escuela Nacional de Bibliotecarios y Archivistas 5 (28) (abril-diciembre, enero-agosto): 21-23.

Gordillo Gordillo, R. A. 1962-1963a. "Palabras del profesor Roberto A. Gordillo". Boletín de la Escuela Nacional de Bibliotecarios y Archivistas 5 (29) (septiembre-diciembre, enero-agosto): 37-42.

Gordillo Gordillo, R. A. 1962-1963b. "Palabras dichas por el C. Roberto A. Gordi1lo, director de esta escuela, en la velada luctuosa en honor del C. Profesor Agustín Hernández Piuki, el 12 de octubre de 1962”. Boletín de la Escuela Nacional de Bibliotecarios y Archivistas 5 (29) (septiembre-diciembre, enero-agosto): 5-6.

Gordillo Gordillo, R. A. 1967a. "El aspecto técnico: la bibliografía nacional”, en Las bibliotecas en la vida nacional: informe final de las Cuartas Jornadas Mexicanas de Biblioteconomía celebradas en la Ciudad de Jalapa, Ver., del 5 al 8 de mayo de 1965, bajo el auspicio de la Universidad Veracruzana, 108-109. México: AMBAC.

Gordillo Gordillo, R. A. 1967b. "Servicios y materiales de lectura para recién alfabetizados", en Las bibliotecas en la vida nacional: informe final de las Cuartas Jornadas Mexicanas de Biblioteconomía celebradas en la Ciudad de Jalapa, Ver., del 5 al 8 de mayo de 1965, bajo el auspicio de la Universidad Veracruzana, 16-29. México: AMBAC.

Gordillo Gordillo, R. A. 1971a. "Estructura, recursos y funcionamiento de las bibliotecas mexicanas". Bibliotecas y Archivos (Escuela Nacional de Biblioteconomía y Archivonomía) 2: 63-101 más una tabla anexa.

Gordillo Gordillo, R. A. 1971b. "Las bibliotecas públicas de la República Mexicana: análisis de su situación actual". La biblioteca: factor esencial de la reforma educativa: informe final de las Quintas Jornadas Mexicanas de Biblioteconomía celebradas en la Ciudad de México, D.F. del 25 al 29 de agosto de 1969 en el auditorio del Instituto Tecnológico Autónomo de México, 31-67. México: AMBAC: ANUIES.

Gordillo Gordillo, R. A. 1974. "Disquisiciones sobre unas normas mínimas para el servicio bibliotecario en escuelas de medicina y otras instituciones dedicadas al estudio de las ciencias de la salud". Bibliotecas y Archivos (Escuela Nacional de Biblioteconomía y Archivonomía) 5: 51-71.

Gordillo Gordillo, R. A. 1975a. "Comentarios sobre la ponencia de la Sra. Ma. Carmen A. de Ciurlizza, intitulada filosofía de los servicios de información”, en VI Jornadas Mexicanas de Biblioteconomía: integración del servicio bibliotecario nacional bibliotecario, Guanajuato, Gto., 17-22 de noviembre, 1974, 191-196. México: AMBAC.

Gordillo Gordillo, R. A. 1975b. "Normas mínimas para el servicio bibliotecario en universidades latinoamericanas". Bibliotecas y Archivos (Escuela Nacional de Biblioteconomía y Archivonomía) 6: 97-146.

Gordillo Gordillo, R. A. 1975c. "Notas preliminares sobre las innovaciones que aparecen en el proyecto de reglamento de la Asociación Mexicana de Bibliotecarios, A.C", en Jornadas mexicanas de biblioteconomía: integración del servicio bibliotecario nacional bibliotecario, Guanajuato, Gto., 17-22 de noviembre, 1974, 469-475. México: AMBAC. 
Gordillo Gordillo, R. A. 1977. "Normas para el establecimiento y sostenimiento de bibliotecas públicas en la República Mexicana”, en VIII Jornadas Mexicanas de Biblioteconomía, del 10 al 6 de mayo de 1977, Guadalajara, Jal.: la problemática de las bibliotecas en México y sus soluciones: memorias, 117-152. México: AMBAC.

Gordillo Gordillo, R. A. 1978. "Normas para el establecimiento y sostenimiento de bibliotecas públicas en la República Mexicana”. Bibliotecas y Archivos (Escuela Nacional de Biblioteconomía y Archivonomía) 9: 9-45.

Gordillo Gordillo, R. A. 1979. "En busca de perfil profesional: disquisiciones obre tres programas de maestría en bibliotecología en México". Ciencia bibliotecaria 3 (2): 91-97.

Gordillo Gordillo, R. A. 1980a. "Evaluación de servicios bibliotecarios", en XI Jornadas Mexicanas de Biblioteconomía, 5 a 9 de mayo de 1980, México, D.F.: el usuario: memorias, 299-302. México: AMBAC.

Gordillo Gordillo, R. A. 1980b. "La administración bibliotecaria". Bibliotecas y Archivos (Escuela Nacional de Biblioteconomía y Archivonomía) 11: 55-89.

Gordillo Gordillo, R. A. 1981. "El planeamiento bibliotecario y los sistemas de toma de decisiones", en V Semana de bibliotecología, del 12 al 16 de octubre de 1981, Centro de Convenciones, Danza del Sol Ajijic, Jalisco: Administración bibliotecaria eficiente: imperativo nacional: memorias, 1-9. Guadalajara: UAG.

Gordillo Gordillo, R. A. 1982. "Los primeros dos años del Colegio Nacional de Bibliotecarios: un informe”. Bibliotecas y Archivos (Escuela Nacional de Biblioteconomía y Archivonomía) 13: 99-116.

Gordillo Gordillo, R. A. 1983a. "Dos puntales para la estructura del servicio bibliotecario mexicano", en XIV Jornadas Mexicanas de Biblioteconomía: 2 A 6 de mayo de 1983, Zacatecas, Zac.: Memorias, 275-284. México: AMBAC.

Gordillo Gordillo, R. A. 1983b. "Reflexiones sobre PRODENASBI y revisión de la propuesta de normas para el establecimiento y sostenimiento de bibliotecas públicas en la República Mexicana”, en XIV Jornadas Mexicanas de Biblioteconomía: 2 a 6 de mayo de 1983, Zacatecas, Zac.: Memorias, 311-326. México: AMBAC.

Gordillo Gordillo, R. A. 1984. "El profesor universitario como usuario y promotor del servicio bibliotecario, análisis de dos casos: el caso sobre el curso de pensamiento bibliotecológico mexicano y el curso historia y filosofía de la ciencia en México”, en Semana de bibliotecología, 7a. ed., 37-50. Memorias. Guadalajara: UAG.

Gordillo Gordillo, R. A. 1985a. "Mesa redonda: comentarios de los expresidentes de la AMBAC", en XV Jornadas Mexicanas de Biblioteconomía, del 30 de abril al 4 de Mayo de 1984, Tlaxcala, Tlax.: memorias, 468-470. México: AMBAC.

Gordillo Gordillo, R. A. 1985b. "El profesor universitario como usuario y promotor del servicio bibliotecario: análisis de dos casos”. Bibliotecas y Archivos (Escuela Nacional de Biblioteconomía y Archivonomía) 16: 177-186.

Gordillo Gordillo, R. A. 1985c. "El usuario marginado: el caso de las escuelas y facultades de psicología de la República Mexicana en relaciones con sus recursos de información”, en XIII Jornadas Mexicanas de Biblioteconomía, mayo 3-7 de 1982, Hermosillo, Son.: Memoria, 447-468. México: AMBAC.

Gordillo Gordillo, R. A. 1985d. "La innovación bibliotecaria y los avances en la enseñanza de la biblioteconomía en México, el caso de Aberytwyth”. Bibliotecas y Archivos (Escuela Nacional de Biblioteconomía y Archivonomía) 16: 127-139.

Gordillo Gordillo, R. A. 1985e. "Profesores para las escuelas mexicanas de biblioteconomía: una prospección”. Bibliotecas y Archivos (Escuela Nacional de Biblioteconomía y Archivonomía) 16: 169-176. 
Gordillo Gordillo, R. A. 1986. "Mesa redonda sobre ética profesional”, en XVI Jornadas Mexicanas de Biblioteconomía, 6 al 10 de mayo de 1985, Pacbuca, Hgo.: memorias, 267-278. México: AMBAC.

Gordillo Gordillo, R. A. 1988. "La misión de las bibliotecas en las zonas fronterizas", en XIX Jornadas Mexicanas de Biblioteconomía, 9 al 11 de marzo de 1988, Villahermosa, Tab.: memorias, 175-186. México: AMBAC.

Gordillo Gordillo, R. A. 1989a. "El usuario mexicano en el año 2000", en XVIII Jornadas Mexicanas de Biblioteconomía, 2 al 6 de marzo de 1987: memorias, 79-84. México: AMBAC.

Gordillo Gordillo, R. A. 1989b. "Legislación bibliotecaria: mesa redonda”, en XVIII Jornadas Mexicanas de Biblioteconomía, 2 al 6 de marzo de 1987: memorias, 173201. México: AMBAC.

Gordillo Gordillo, R. A. 1993. "El robo, la mutilación y la ocultación de libros de texto en las bibliotecas universitarias mexicanas: propuesta para el estudio científico del problema", en Memorias de las XXII Jornadas Mexicanas de Biblioteconomia, 77-93. México: Gobierno del Estado de Chiapas.

Gordillo Gordillo, R. A. 1994. "El papel de la biblioteca pública mexicana ante la nueva Ley General de Educación”, en XXIV Jornadas Mexicanas de Biblioteconomía: memoria, 13, 14 y 15 de septiembre de 1993, 49-69. Guadalajara, México: Secretaría de Cultura del Estado de Jalisco, Universidad de Guadalajara, AMBAC.

Gordillo Gordillo, R. A. 1996. "Reflexiones sobre el usuario marginado", en Memorias de las XXV Jornadas Mexicanas de Biblioteconomia, Puerto Vallarta, Jalisco, del 2 al 4 de mayo de 1994, 247-257. México: AMBAC.

Gordillo Gordillo, R. A. 1998. "La afiliación a las asociaciones de bibliotecarios: en caso de la pertinencia y de la pertenencia", en Colegio Nacional de Bibliotecarios, A. C. El significado del bibliotecario: una antología para el futuro profesional, 15-20. México: CNB. Fecha de consulta: 20 de agosto de 2016. http://www.cnb. org.mx/publicaciones/Voutssas-Significado_del_Bibliotecario.pdf

Gordillo Gordillo, R. A. 2004. "Mensaje de Roberto Gordillo Gordillo”, en Vinculación de las bibliotecas y la academia: un esfuerzo compartido: memoria del IX Coloquio Internacional de Bibliotecarios, 177-181. Guadalajara, México: Universidad de Guadalajara.

Gordillo Gordillo, R. A. 2006. Una visión prospectiva de la profesión bibliotecaria en México, disco compacto. México: CNB.

Parsons, M. D. y R. A. Gordillo Gordillo. 1958. Directorio de bibliotecas de la Ciudad de México $=$ Directory of México City Libraries. México: Mexico City College.

Para citar este texto:

Martínez Musiño, Celso. 2018. "Roberto Gordillo: planificador y líder, formador de profesionales y organizador gremial”. Investigación Bibliotecológica: archivonomía, bibliotecología e información 32 (74): 123-143.

http://dx.doi.org/10.22201/iibi.24488321xe.2018.74.57914 